\title{
Microarray Shared Resource
}

National Cancer Institute

\section{Source}

National Cancer Institute. Microarray Shared Resource. NCI Thesaurus. Code C39458.

The Microarray Shared Resource supports Cancer Center research activity performing gene expression profiling in which the activity of many thousands of genes is measured simultaneously. The Resource provides biochemical, hybridization, and scanning services, genome-wide SNP analysis and resequencing analysis, consultation in experimental design and data mining. 